\title{
EDITORIAL
}

\section{Neurobiology of suicidal behavior in combat veterans}

\author{
Leo Sher ${ }^{1,2}$ iD \\ ${ }^{1}$ James J. Peters Veterans' Administration Medical Center, Bronx, NY, USA. ${ }^{2}$ Department of Psychiatry, Icahn School of Medicine at Mount \\ Sinai, New York, NY, USA.
}

Wars have been and remain a constant companion of humans throughout history, and nothing seems to suggest that a time free of wars will soon come to be reality. Multiple studies and observations suggest that combat veterans are at increased risk for attempting and dying by suicide. ${ }^{1}$ Suicide among combat veterans is an important issue across the world. In 2014, more than 7,400 U.S. military veterans died by suicide, accounting for $18 \%$ of all deaths by suicide in the U.S., notwithstanding the fact that veterans make up only $8.5 \%$ of the U.S. population. ${ }^{1}$

These greater rates of suicide may be related to the fact that military servicemembers are in danger of death each day and may be desensitized to it. Also, combat veterans are subjected to various chemical, physical, and environmental factors that may alter the brain. A psychobiological condition of combat veterans is dissimilar from a psychobiological state of non-combat veterans and civilians. In many ways that influences their suicide risk; combat veterans are not like the general population. The exceptionality of the experience of military combat as a contributor to suicidal behavior merits specific investigation of neurobiological factors that may be associated with suicidality in combat veterans. In this article, I will discuss our and some other studies and suggest that more research into the neurobiology of suicidal behavior among combat veterans is needed.

There are very few studies of the neurobiology of suicidality among combat veterans. We have performed investigations of the neurobiology of suicidal behavior in combat military veterans. ${ }^{2,3}$ We compared combat veterans with or without a history of post-deployment suicide attempts, and found that suicide attempters had higher suicidal ideation scores in comparison to non-attempters. Our studies indicate that combat veterans with a history of attempting suicide remain at elevated suicide risk long after the attempt.

In our studies, suicide attempters had significantly lower blood levels of dehydroepiandrosterone (DHEA) and dehydroepiandrosterone sulfate (DHEA-S) compared to non-attempters. ${ }^{2}$ Decreased amounts of DHEA and DHEA-S may result in elevated susceptibility to neurotoxic influences of stress and contribute to the neurobiology of stress-related disorders and suicidality. We observed that DHEA-S concentrations correlated negatively with suicidal ideation scores in suicide non-attempters, but not in attempters. Possibly, as psychiatric conditions progress, the level of DHEA-S decreases; hence, the neuroprotection provided by DHEA-S is considerably reduced or lost, leading to increased suicidality.

We have also examined a relation between two endogenous opioids, anandamide (AEA) and 2-arachidonoylglycerol (2-AG), with suicidal behavior and suicidality in combat veterans. ${ }^{3}$ We were interested in the role of endogenous cannabinoids in the neurobiological mechanisms of suicidality among combat veterans because changes in endocannabinoid signaling may play a role in the development of stress-related psychiatric conditions, including mood disorders and posttraumatic stress disorder (PTSD). Our interest was also related to the fact that veterans often use cannabis, and that an association has been observed between use of cannabis among Iraq/ Afghanistan-era veterans and suicidal behavior. We found that, controlling for gender, 2-AG blood concentrations were greater among suicide attempters compared to non-attempters. We also found that AEA blood concentrations correlated negatively with suicidal ideation scores among suicide attempters, but not among non-attempters. AEA appears to reduce suicidality among suicide attempters, i.e., AEA plays a part in the secondary prevention of suicide attempt. AEA may diminish suicidality because the endocannabinoid system restricts activation of the stress reaction by way of disseminated actions in limbic and hypothalamic circuits in the brain. Our research suggests that clinically detected dissimilarities between combat military veterans with vs. without a history of postdeployment suicide attempts may have a neurobiological origin.

Other researchers have also studied the neurobiology of suicide among combat veterans. One research group compared platelet serotonin concentrations in five groups of male individuals: suicidal and non-suicidal combat veterans with combat-related PTSD, suicidal and nonsuicidal psychiatric patients without PTSD, and healthy controls. ${ }^{4}$ Platelet serotonin concentrations were significantly lower in suicidal PTSD and non-PTSD patients compared to non-suicidal PTSD and non-PTSD patients
Correspondence: James J. Peters Veterans' Administration Medical Center, 130 West Kingsbridge Road, Bronx, NY 10468, USA.

E-mail: Leo.Sher@mssm.edu

Submitted Jan 28 2020, accepted Feb 06 2020, Epub Apr 032020.
How to cite this article: Sher L. Neurobiology of suicidal behavior in combat veterans. Braz J Psychiatry. 2020;42:465-466. http://dx.doi. org/10.1590/1516-4446-2020-0877 
and to healthy controls. Another study showed that, relative to combat veterans without suicidal ideation, combat veterans with suicidal ideation demonstrated less activation in a large region across the lateral prefrontal cortex and cingulate cortex, as well as the inferior temporal cortex, in response to interference demands. ${ }^{5}$

Current attempts at suicide prevention among military veterans are hampered by the deficiency of knowledge concerning the neurobiological mechanisms of suicidal behavior in this population. The lack of biological tests to detect suicidality and the lack of effective medications to treat it are two major issues in suicide research and prevention. Studies of the neurobiology of suicidal behavior among combat veterans may lead to improvement in detection of suicidality and potential new pharmacological targets for the treatment of suicidality in this population. Possibly, studies should extend beyond the influence of psychiatric illness and try to find other factors that contribute to suicidality in combat veterans. We will not be able to reduce suicides in combat veterans until we have a sound understanding of the psychobiology of this phenomenon.

\section{Disclosure}

The author reports no conflicts of interest.

\section{References}

1 VA Suicide Prevention Program. Facts about veteran suicide. 2016 Jul [cited 2020 Mar 2]. gov/opa/publications/factsheets/Suicide Prevention_FactSheet_New_VA_Stats_070616_1400.pdf

2 Sher L, Flory J, Bierer L, Makotkine I, Yehuda R. Dehydroepiandrosterone and dehydroepiandrosterone sulfate levels in combat veterans with or without a history of suicide attempt. Acta Psychiatr Scand. 2018;138:55-61.

3 Sher L, Bierer LM, Flory J, Hill MN, Makotkine I, Yehuda R. Endogenous cannabinoid levels and suicidality in combat veterans. Psychiatry Res. 2020;287:112495.

4 Kovacic Z, Henigsberg N, Pivac N, Nedic G, Borovecki A. Platelet serotonin concentration and suicidal behavior in combat related posttraumatic stress disorder. Prog Neuropsychopharmacol Biol Psychiatry. 2008;32:544-51.

5 Bomyea J, Stout DM, Simmons AN. Attenuated prefrontal and temporal neural activity during working memory as a potential biomarker of suicidal ideation in veterans with PTSD. J Affect Disord. 2019;257:607-14. 